\title{
PUFA-synthase-specific PPTase enhanced the polyunsaturated fatty acid biosynthesis via the polyketide synthase pathway in Aurantiochytrium
}

\author{
Sen Wang ${ }^{1 \dagger}$, Chuanzeng Lan ${ }^{1,2 \dagger}$, Zhuojun Wang ${ }^{1,2}$, Weijian Wan ${ }^{1}$, Qiu Cui ${ }^{1{ }^{*}}$ and Xiaojin Song ${ }^{1 *}$ (i)
}

\begin{abstract}
Background: Phosphopantetheinyl transferase (PPTase) can change the acyl-carrier protein (ACP) from an inactive apo-ACP to an active holo-ACP that plays a key role in fatty acids biosynthesis. Currently, the PPTase has been proved to be involved in the biosynthesis of polyunsaturated fatty acids (PUFAs) via a polyketide synthase (PKS) pathway in Thraustochytrids, while its characteristics are not clarified.

Results: Here, the heterologous PPTase gene ( $\mathrm{faE}$ ) from bacteria was first co-expressed with the PKS system (orfA-orfC) from Thraustochytrid Aurantiochytrium. Then, a new endogenous PPTase ( $p p t \_a$ ) in Aurantiochytrium was identified by homologous alignment and its function was verified in E. coli. Moreover, the endogenous ppt_a was then overexpressed in Aurantiochytrium, and results showed that the production and proportion of PUFAs, especially docosahexaenoic acid (DHA), in the transformant SD1 16::PPT_A were increased by $35.5 \%$ and $17.6 \%$, respectively. Finally, higher DHA and PUFA proportion (53.9\% and 64.5\% of TFA, respectively) were obtained in SD1 16::PPT_A using a cerulenin feeding strategy.
\end{abstract}

Conclusions: This study has illustrated a PUFAs-synthase-specific PPTase in PKS system and provided a new strategy to improve the PUFA production in Thraustochytrids.

Keywords: Aurantiochytrium, DHA, Thraustochytrids, Polyketide synthase, PPTase

\section{Background}

Polyunsaturated fatty acids (PUFAs), especially docosahexaenoic acid (DHA, 22:6 $\omega 3$ ) and eicosapentaenoic acid (EPA, 20:5 $\omega 3$ ), are rapidly gaining attention, due to their beneficial effects in the cognitive development

\footnotetext{
*Correspondence: cuiqiu@qibebt.ac.cn; songxj@qibebt.ac.cn

†'Sen Wang and Chuanzeng Lan have contributed equally to this work

${ }^{1}$ CAS Key Laboratory of Biofuels, Shandong Provincial Key Laboratory of Energy Genetics, Shandong Engineering Laboratory of Single Cell Oil, Qingdao Engineering Laboratory of Single Cell Oil, Qingdao Institute of Bioenergy and Bioprocess Technology, Chinese Academy of Sciences, No.189 Songling Road, Laoshan District, Qingdao 266101, Shandong, China

Full list of author information is available at the end of the article
}

of infants and their use to reduce the risk of hypertension, cardiovascular diseases, inflammation, and certain cancers [1-4]. Currently, the major commercial source of PUFAs is fish oil; however, several factors such as the reduction of marine fish source, the increasing environmental pollution, and undesirable fishy flavor limit the supply of high-quality PUFAs $[5,6]$. Therefore, alternative sources of high-quality PUFAs, especially DHA and EPA, have drawn increasing amounts of attention. Heterotrophic thraustochytrids, are capable of accumulating large of lipids, have been increasing embraced by the market $[7,8]$.

As it is known, PUFAs are synthesized by two pathways, the aerobic desaturase/elongase pathway and the

c) The Author(s) 2020. This article is licensed under a Creative Commons Attribution 4.0 International License, which permits use, sharing, adaptation, distribution and reproduction in any medium or format, as long as you give appropriate credit to the original author(s) and the source, provide a link to the Creative Commons licence, and indicate if changes were made. The images or other third party material in this article are included in the article's Creative Commons licence, unless indicated otherwise in a credit line to the material. If material is not included in the article's Creative Commons licence and your intended use is not permitted by statutory regulation or exceeds the permitted use, you will need to obtain permission directly from the copyright holder. To view a copy of this licence, visit http://creativecommons.org/licenses/by/4.0/. The Creative Commons Public Domain Dedication waiver (http://creativecommons.org/publicdomain/zero/1.0/) applies to the data made available in this article, unless otherwise stated in a credit line to the data. 
anaerobic polyketide synthase (PKS) pathway [9-11]. Compared with the desaturase/elongase pathway, PKS pathway directly synthesizes the PUFAs from acetyl-CoA and malonyl-CoA substrates with a few intermediates. The PKS pathway is catalyzed by an enzyme complex, and the gene codings for the members of this complex are composed of three or four subunits and possess similar domain structures including $\beta$-ketoacyl synthase (KS), chain length factor (CLF), malonyl-CoA transacylase (MAT), acyl-carrier protein (ACP), ketoacyl reductase (KR), acyltransferase (AT), enoyl reductase (ER), and dehydratase (DH) domains (Fig. 1) [8, 12-14]. In addition to the above domains, a phosphopantetheine transferase (PPTase) is also reported to be essential for PUFA synthesis [15]. ACP domain in PKS needs a posttranslational modification to become enzymatically active. PPTase transfers the pantetheine moiety from Coenzyme A to a conserved serine residue of an inactive carrier protein to produce its active form $[16,17]$. The gene encoding that PPTase (PfaE) has been identified in several bacterial PKS systems, such as Shewanella oneidensis [18] and Moritella marina [19], and has been proved to be essential for PUFA synthesis. The putative PPTase encoding gene was also identified in the Thraustochytrid species, such as Aurantiochytrium limacinum [20] and Hondaea fermentalgiana [7]; however, its characteristics are not clearly clarified.

PPTases can be subdivided into three groups according to their primary sequence and substrate specificity [19, $21,22]$. The name-giving prototype of the first group is AcpS from Escherichia coli. The AcpS-type PPTases are 120-140 amino acids in size, act as homotrimers, are associated with primary metabolism in prokaryotes, and accept only ACPs from FAS and ACPs of type II PKS. The second group of PPTases, which can be found in eukaryotes, represents integrated domains of their cognate FAS.
PPTases of the third, Sfp-type PPTases, are about twice the size of AcpS, have an extraordinarily broad substrate spectrum, and are active as monomers. Sfp has proven to recognize every carrier protein $(\mathrm{CP})$ tested so far, including PCPs of NRPSs and ACPs of FASs and PKSs $[16,19]$.

Heterotrophic thraustochytrid, Aurantiochytrium sp., has a strong ability to produce biomass and lipid. Particularly, it can produce large amounts of DHA, which comprises nearly $50 \%$ of the total fatty acid (TFA) proportion [23-25]. Therefore, Aurantiochytrium has become one of the major alternative sources for commercial DHA production.

It is well known that there are two fatty acid pathways in Aurantiochytrium [4, 26]. The conventional fatty acid synthase (FAS) pathway is mainly responsible for the synthesis of saturated fatty acids (SFAs), while PUFAs including DHA and docosapentaenoic acid (DPA) in Aurantiochytrium are synthesized via the PKS pathway $[4,27]$. This PKS system has been confirmed to contain three genes, called $\operatorname{orf} A$, $\operatorname{orf} B$, and $\operatorname{orfC}$ (Fig. 1). However, no PPTase gene or PPTase function domain was contained in this PKS system [13, 28].

In the present work, we first confirmed that PPTase was involved in and essential for the PKS system in Aurantiochytrium and that the efficiency of DHA synthesis was influenced by overexpression of PPTase. Then, a new endogenous PPTase was identified and overexpressed in Aurantiochytrium sp. SD116 to strengthen the PKS system resulting in the improvement of DHA production. The proportion of PUFAs, especially the DHA proportion, had significantly increased in the recombinant SD116::PPT_A strain. The PUFAs proportion was further enhanced by depressing the FAS pathway. Based on this study, a PUFAs-synthase-specific PPTase in PKS system was identified and a new strategy for high PUFA production in Aurantiochytrium was provided.

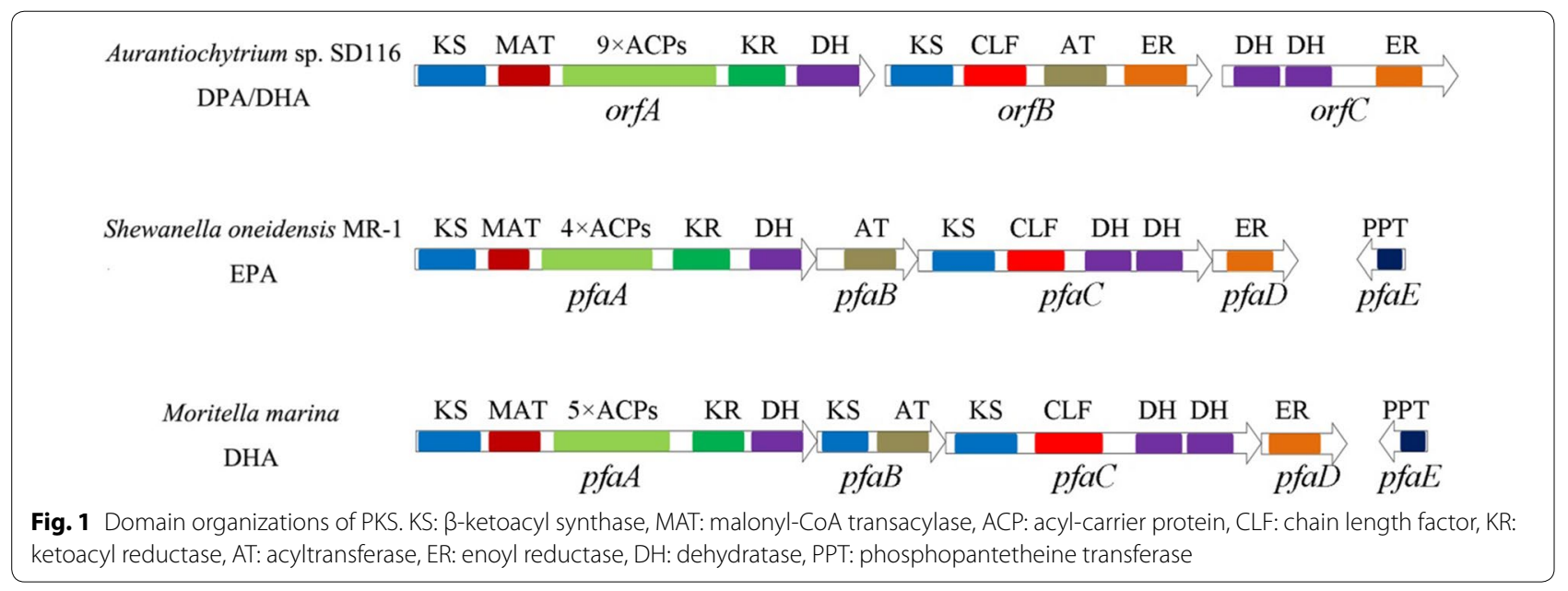




\section{Results and discussion}

\section{PPTase is essential for the PKS system}

In thraustochytrid Aurantiochytrium, the so-called PKS, can anaerobically synthesize PUFAs de novo. The PKS organization consists of three subunits OrfA, OrfB, and OrfC (Fig. 1). To verify all components of PKS in Aurantiochytrium, orfA, orfB, and orfC were cloned and successfully co-expressed in E. coli. GC-MS was used to analyze the lipid profiles of $E$. coli, and the result showed that no DHA was produced by the recombinant strain

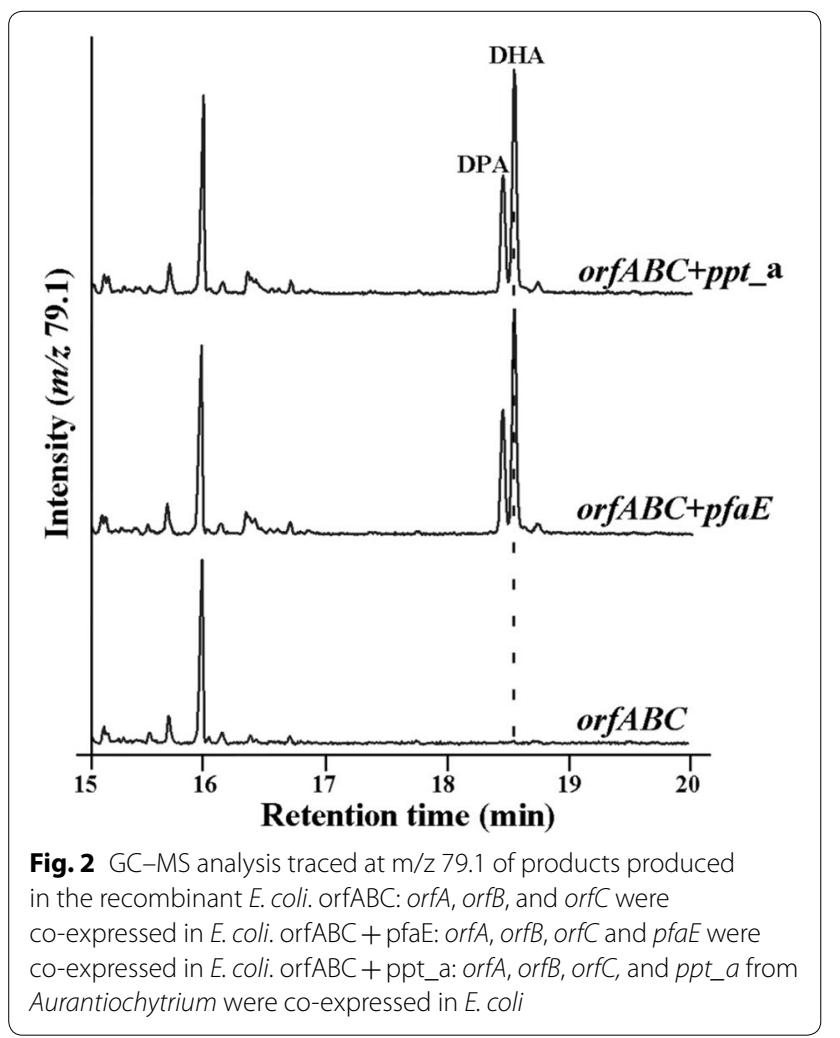

orf $A B C$ (Fig. 2), suggesting that the heterologous expression of PKS system is not intact. The PKS organization in Aurantiochytrium possesses AT, multiple ACPs, MAT, $\mathrm{KS}, \mathrm{KR}, \mathrm{DH}, \mathrm{ER}$, and CLF domains, while no PPTase encoding gene or PPTase domain was identified. The previous studies showed that PPTase is essential for PUFA production in several marine bacteria, and the recombinant product of EPA and DHA in E. coli was achieved using the intact PKS gene cluster which contains the PPTase encoding gene from Shewanella and Moritella marina, respectively $[29,30]$. Therefore, we deduced that the PPTase may be also essential for the PKS system of Aurantiochytrium. pfaE gene, which encodes a PPTase in Shewanella, was cloned and co-expressed with $\operatorname{orf} A$, orfB, and orfC in E. coli, and the result showed that the recombinant DHA and DPA were produced (Fig. 2). Based on this result, we confirmed that PPTase is involved in and essential for the PKS system in Aurantiochytrium.

As functional expression of the PKS requires posttranslational activation by a PPTase, overexpressing PPTase may be beneficial for PUFA synthesis in Aurantiochytrium. Then, the pfaE gene from Shewanella was overexpressed in Aurantiochytrium to attempt to improve the DHA production. As shown in Additional file 1 , the plasmid pWE-PfaE containing the $p f a E$ expression cassette driven by a tubulin promoter (Ptub) and actin transcription terminator (Tactin) was transformed into Aurantiochytrium sp. SD116, and the correct transformants named SD116::PfaE were screened by the selection stress of zeocin and further confirmed by genomic PCR. A 0.9-Kbp DNA band was detected in the transformant, but not in SD116 (Additional file 2), suggesting that $\mathrm{p} f a E$ was successfully inserted in SD116.

To demonstrate the effects of $p f a E$ overexpression, the biomass, lipid accumulation, and fatty acid composition were analyzed. As shown in Fig. 3, the final biomass (dry cell weight) of the transformant SD116::PfaE was $21.6 \%$
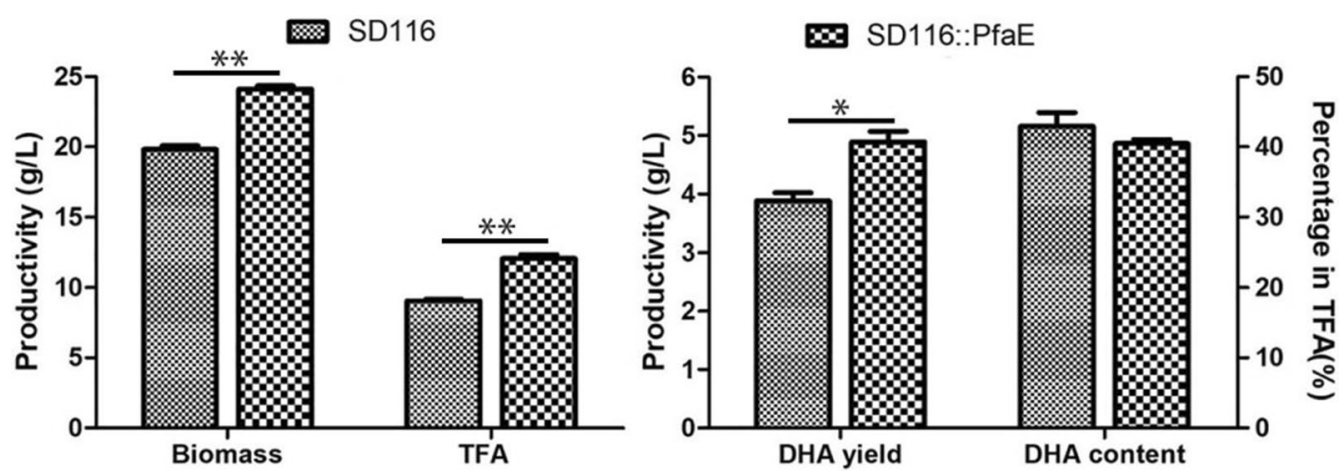

Fig. 3 Fermentation profiles in strains SD116 and SD116::PfaE. Biomass and the total fatty acid production in SD116 and SD116::PfaE (left). DHA production and DHA proportion in SD116 and SD116::PfaE (right). ${ }^{*} p<0.01 .{ }^{*} p<0.05$ 
$(p<0.001)$ higher than that of SD116 at the stationary phase (96 h). In addition, the total fatty acid (TFA) production and the DHA production of the transformant SD116::PfaE were $33.4 \%(p<0.001)$ and $25.9 \%(p<0.05)$ higher than those in SD116, respectively. However, the DHA proportion (percentage in TFAs) in SD116::PfaE is almost the same to that in SD116 (Fig. 3). Thus, it is evident that overexpression of the PPTase encoding by $p f a E$ in Aurantiochytrium can significantly improve its ability of fatty acid synthesis (Additional file 3). From these results, we concluded that the PfaE, an Sfp-type enzyme from Shewanella, had a relatively broad substrate spectrum which was consistent with the results inferred from its primary structure [17], and it could activate the acylcarrier protein both from the FAS and PKS pathway in Aurantiochytrium.

\section{An endogenous PPTase was identified in Aurantiochytrium}

The previous study found that substitution of the PPTase gene from the heterologous species did not influence the function of the PKS pathway producing DHA, but led to different DHA yields [17], suggesting that PPTases are also required specifically to recognize the structure of substrates. There are four repeated ACP domains (for $S$. oneidensis.) or nine (Aurantiochytrium sp. SD116) are integrated in the large multifunctional PfaA (or OrfA) product as substrates of PPTases (Fig. 1). Therefore, although different PPTases could lead to PUFA synthesis, they may have a very strict specificity for efficiently activating their substrates. A PPTase which can specifically recognize the nine repeated ACP domains may be better for PUFAs synthesis in Aurantiochytrium.

The recombinant DHA was produced in E. coli after the $p f a E$ was co-expressed with the PKS system of Aurantiochytrium, so we deduced that there might be a PPTase in Aurantiochytrium involved in the synthesis of PUFAs. A blast search using S. oneidensis MR-1 PfaE as probe from the Aurantiochytrium sp. SD116 genome yielded one contig encoding a putative PPTase. The putative PPTase (named as PPT_A) contains 271aa (30.5 kDa) with a $\mathrm{pI}$ of 5.22, and it exhibits a relatively low similarity (only 24.19\%) to the PfaE. To confirm that the PPT_A has PPTase activity, $p p t a$ was cloned and co-expressed with $\operatorname{orfA}$, orfB, and $\operatorname{orfC}$ in E. coli. As shown in Fig. 2, the recombinant $E$. coli could synthesize DHA. Thus, it is confirmed that PPT_A is a PPTase which can activate the biosynthesis process of PUFAs.

Alignment of representative PfaE [PfaE from S. oneidensis (PfaE_S) and M. marina (PfaE_M)], PPTase (PPT_M from $A$. limacinum ATCC MYA-1381, PPT_H from $H$. fermentalgiana.), and PPT_A protein sequences revealed the existence of $\mathrm{P} 1, \mathrm{P} 2$, and $\mathrm{P} 3$ domains, and the P1 domain was recognized separately as two subdomains of P1a and P1b (Additional file 4). These conserved amino acid domains were defined previous $[19,21]$. P2 and P3 are domains participating in $\mathrm{Mg}^{2+}$ binding, and P1 (both P1a and P1b) and P3 are involved in substrate (coenzyme A) binding and catalysis [31, 32]. PfaE for EPA and DHA had another conserved sequence of $\mathrm{L} / \mathrm{VRxL} /$ VLS (P0) [19]. The P0 domain and P1a and P1b domains were predicted to be associated with recognition of the specific tertiary structure of the substrates carrying repeated ACP domains of the PfaA product. However, no similar P0 sequence was identified in PPT_A. Peng et al. alignment of four representative $\mathrm{PfaE}$ protein sequences revealed that the PO domain is not conserved among these four PfaE sequences [17]. The substrate of PPT_A contains nine repeated ACP domains, while the PfaE from S. oneidensis and M. marina are required to recognize four and five repeated ACP domains, respectively. Therefore, we deduced that the P0 sequences might be conserved for the structure of substrates.

\section{PPT_A is a PUFA-synthase-specific PPTase in the PKS system of Aurantiochytrium}

The stated result demonstrated that PPT_A is involved in the PUFA biosynthesis (Fig. 2). However, the transcription level of ppta in Aurantiochytrium sp. SD116 is only $4 \%$ of that of fas gene at the logarithmic phase of growth, which is obviously lower than other fatty acid synthesis genes (Fig. 4a). Therefore, we deduced that increasing the PPTase activity may improve the lipid and DHA production in Aurantiochytrium. Therefore, the $p p t a$ gene was overexpressed in vivo. The expression cassette containing $p p t a a$ and $z e o^{\mathrm{R}}$ resistance gene was transformed into Aurantiochytrium via electroporation to obtain SD116::PPT_A strain (Additional file 5). The correct transformants were screened by the selection stress of zeocin and further confirmed by genomic PCR. A $0.9-\mathrm{K}$ bp DNA band was detected in the PCR with the SD116::PPT_A, but not with the wild-type SD116 (Additional file 6), suggesting that $p p t \_a$ was successfully inserted in SD116.

To further characterize SD116::PPT_A transformant, both the cell biomass and fatty acid profiles were detected. As shown in Fig. 5, the final biomass and TFA production of SD116::PPT_A were $6.1 \%(p<0.01)$ and $15.2 \%(p<0.05)$ higher than that of SD116, respectively. These results suggest that overexpression of PPT_A can improve the cell growth and lipid synthesis in Aurantiochytrium. In addition to the increased biomass and TFA production, the DHA production and DHA proportion of SD116::PPT_A were $35.5 \%(p<0.001)$ and $17.6 \%(p<0.001)$ higher than that of SD116, respectively (Fig. 5a). Interestingly, although the biomass and TFA production were increased, the total saturated fatty acid 

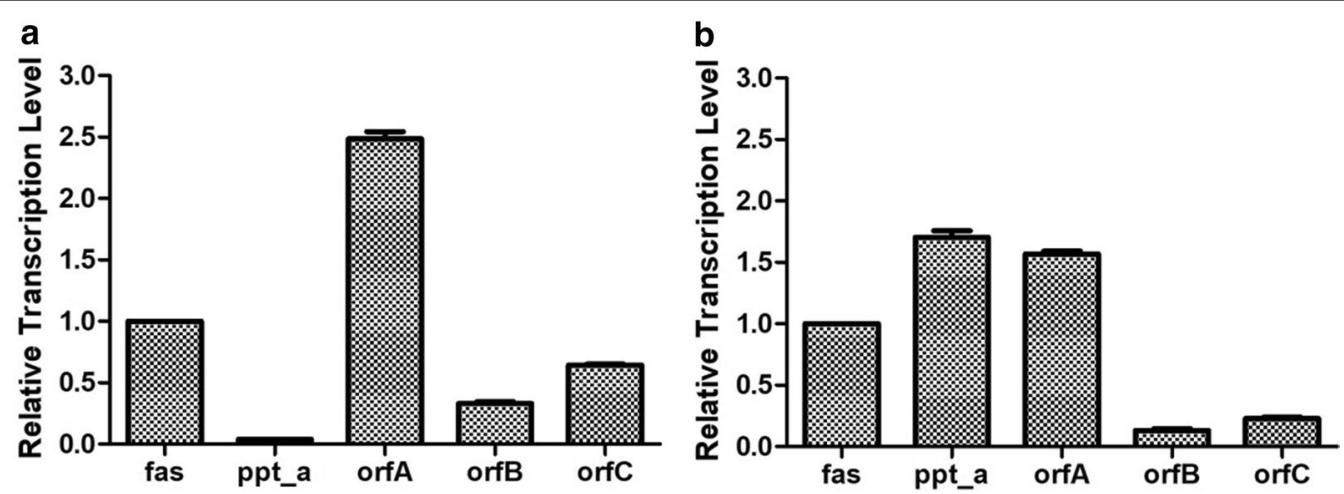

Fig. 4 The relative transcription levels of the fatty acid synthesis genes in strain SD116 (a) and SD116::PPT_A (b). fas gene was used as the contrast in each strains

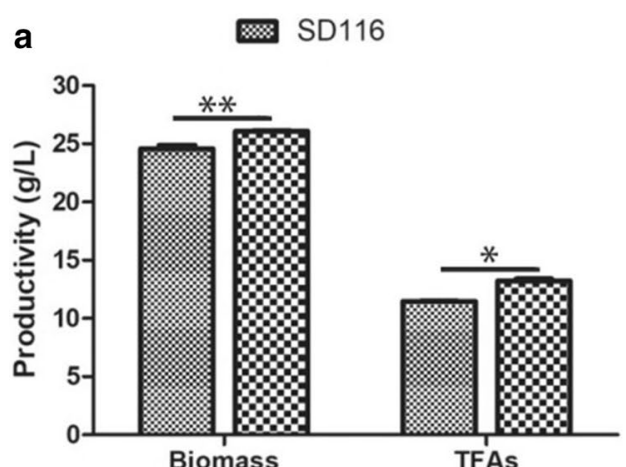

Biomass

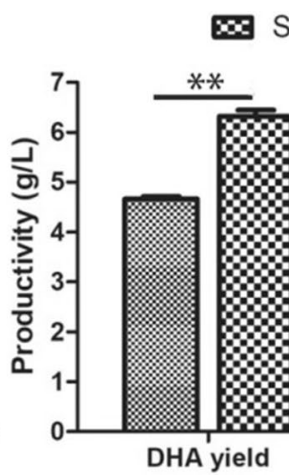

\section{SD116::PPT_A}

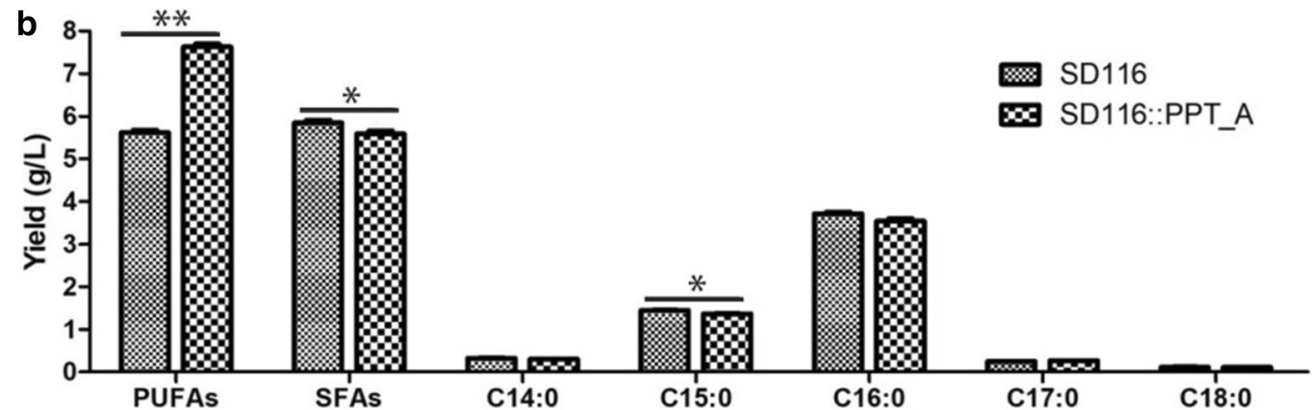

Fig. 5 Fermentation profiles in strains SD116 and SD116::PPT_A. a Biomass and the total fatty acid production in SD116 and SD116::PPT_A (left). DHA production and DHA proportion in SD116 and SD116::PPT_A (right). b Saturated fatty acid (SFA) yields in strains SD116 and SD116::PPT_A. ${ }^{* *} p<0.01 .{ }^{*} p<0.05$

(SFA) production of SD116::PPT_A is 95.5\% $(p<0.05)$ of that of SD116 (Fig. 5b), suggesting that overexpression of PPT_A has little effect on the SFAs pathway. From these results, we concluded that the newly discovered endogenous PPT_A can specifically activate the PUFA biosynthesis pathway. Due to the successful overexpression of the ppt_a gene, the transcription level of $p p t \_a$ in SD116::PPT_A was 1.70-fold of that of fas gene, while the transcription level ratio $\left(l_{p p t a} a\right.$ vs $l_{f a s}$, the transcription level of fas gene was used as the control, because SFAs' production had little effect by overexpressing the $p p t \_a$ in Aurantiochytrium) was 42.5 times that of wild type (Fig. 4b). Moreover, the relative transcription ratios of $\operatorname{orfA}$, orfB, and orfC in SD116::PPT_A were significantly decreased. At the same time, the expression levels of these genes in transformant containing empty plasmid did not change significantly compared to wild-type strain (Additional file 7). Therefore, we deduced that the newly 
discovered endogenous PPT_A played a key role in promoting the efficiency of PUFAs biosynthesis.

\section{Enhancement of DHA proportion using the cerulenin feeding strategy}

The endogenous PPT_A can specifically activate the PUFA biosynthesis pathway and has little effect on SFA synthesis pathway. Thus, inhibition of the SFA biosynthesis pathway might further improve the DHA proportion and production. Cerulenin is a fungal antibiotic that irreversibly inhibits the activity of KS domain [33]. Previous study showed that lower concentrations of cerulenin inhibited SFA synthesis pathway, and higher concentrations blocked the PUFA synthesis pathway in Schizochytrium [34]. Therefore, $0.1 \mathrm{mg} / \mathrm{L}$ cerulenin was used to enhance PKS pathway in Aurantiochytrium. As shown in Fig. 6a, the final biomass and TFA production were decreased by $8.4 \%(p<0.001)$ and $18.4 \%(p<0.001)$ in SD116::PPT_A, respectively, after addition of cerulenin in medium. The DHA production was decreased by $8.0 \%(p<0.05)$ when SD116::PPT_A was grown with cerulenin; however, the DHA and PUFAs proportion were increased by $12.7 \%(p<0.001)$ and $11.3 \%(p<0.001)$, respectively (Fig. 6b). Although cerulenin inhibits the final biomass and TFA production, SD116::PPT_A could produce higher DHA proportion (53.9\%) which is beneficial to a high-quality DHA product. As shown in Fig. 6c, the yields of DHA and PUFAs in SD116::PPT_A were $242.5 \pm 7.0 \mathrm{mg} / \mathrm{g} \mathrm{dcw}$ (dry cell weight) and $292.5 \pm 3.8 \mathrm{mg} / \mathrm{g} \mathrm{dcw}$, respectively. Moreover, these percentages were not significantly changed after addition of cerulenin $(243.7 \pm 0.9 \mathrm{mg} / \mathrm{g} \mathrm{dcw}$ and $290.3 \pm 1.8 \mathrm{mg} / \mathrm{g}$ $\mathrm{dcw}$, respectively), suggesting that cerulenin has little effect on PKS system. The percentage of SFA in SD116::PPT_A decreased by $24.7 \%(p<0.001)$ after addition of cerulenin in medium, suggesting that cerulenin inhibited the SFA synthesis pathway more efficiently. Based on these results, we confirmed that low concentrations of cerulenin could selectively inhibit the SFA synthesis pathway in Aurantiochytrium SD116::PPT_A.

Both PKS and FAS systems use the same substrate to synthesize fatty acids. Here, we found that inhibiting the FAS pathway could improve the DHA proportion in Aurantiochytrium. A high-quality DHA product from SD116::PPT_A was obtained with the cerulenin strategy. However, addition of cerulenin is not conductive to the industrial production of DHA. Further study to downregulate gene expression of fas by genetic engineering technology will provide more advantages for DHA industrial production.

\section{Conclusions}

In this study, a new endogenous PPTase (PPT_A) was identified and proved to be essential to synthesize PUFAs in thraustochytrids Aurantiochytrium. Furthermore, the DHA and PUFA proportion in PPT_A overexpressed strain can reach to $53.9 \%$ and $64.5 \%$ in TFA, respectively. This study has first identified a PUFAs-synthase-specific PPTase in PKS system of Aurantiochytrium and provided
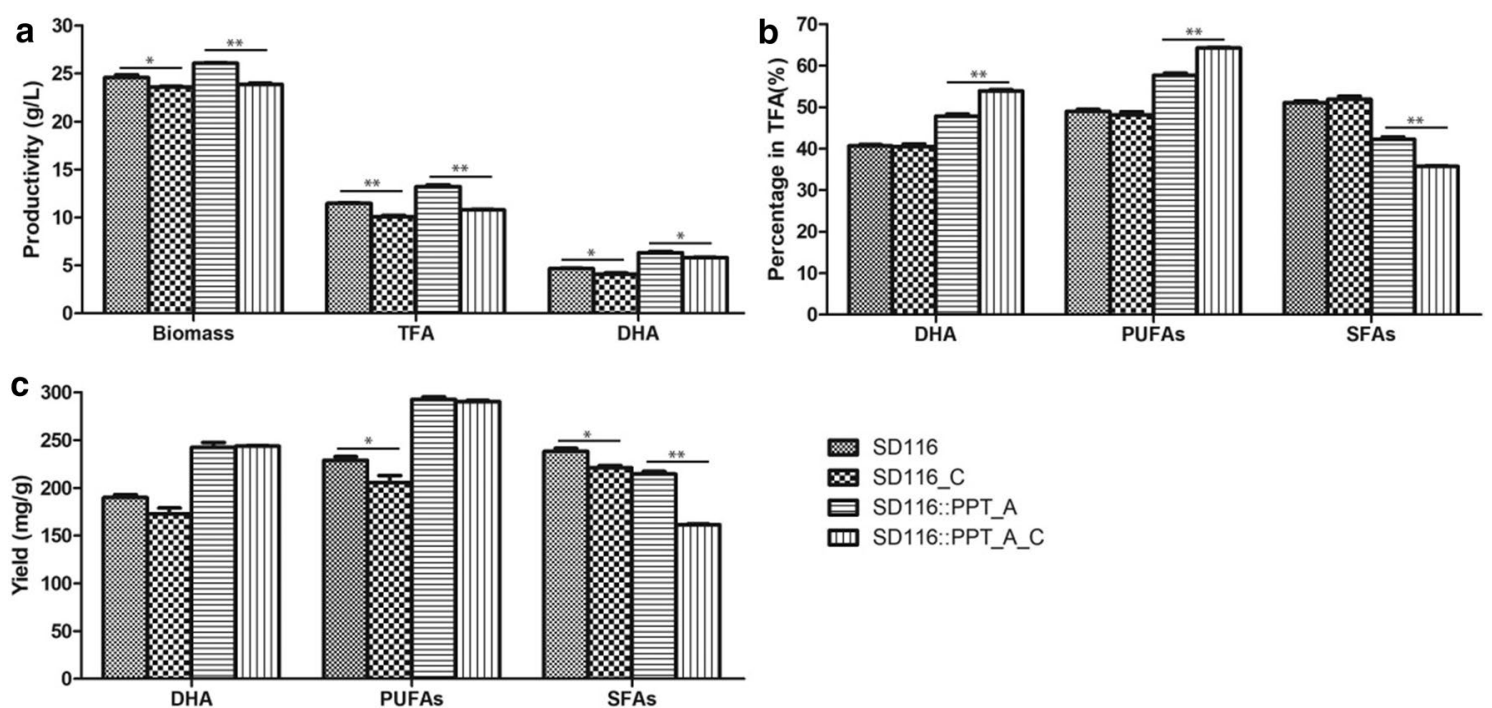

Fig. 6 Fermentation profiles of strains SD116 and the mutant SD1 16::PPT_A upon adding 0.1 mg/L cerulenin (named as SD116_C and SD116::PPT_A_C, respectively). a The final biomass, TFA production, and DHA production in SD116, SD116_C, SD116::PPT_A, and SD116::PPT_A_C. b The DHA, PUFAs, and SFAs' proportion (percentage in TFA) in SD1 16, SD116_C, SD1 16::PPT_A, and SD116::PPT_A_C. c The percentage of DHA, PUFAs, and SFAs (mg/g dcw) in SD116, SD116_C, SD116::PPT_A, and SD116::PPT_A_C. ${ }^{*} p<0.01 .{ }^{*} p<0.05$ 
a new strategy to improve the PUFA production in Thraustochytrids.

\section{Methods}

\section{Strains and cultivation}

Aurantiochytrium sp. SD116 was cultivated in a seed liquid medium containing $30 \mathrm{~g} / \mathrm{L}$ glucose, $10 \mathrm{~g} / \mathrm{L}$ yeast extract, and $10 \mathrm{~g} / \mathrm{L}$ artificial sea salt. The strain was cultivated at $25^{\circ} \mathrm{C}$ and shaken at $200 \mathrm{rpm}$ for $48 \mathrm{~h}$, and then, $2 \%(\mathrm{v} / \mathrm{v})$ of cultures were transferred into fermentation medium containing $60 \mathrm{~g} / \mathrm{L}$ glucose, $20 \mathrm{~g} / \mathrm{L}$ yeast extract, and $15 \mathrm{~g} / \mathrm{L}$ sea salt. The screening medium, containing $30 \mathrm{~g} / \mathrm{L}$ glucose, $10 \mathrm{~g} / \mathrm{L}$ yeast extract, $5 \mathrm{~g} / \mathrm{L}$ artificial sea salt, $1.5 \%$ agar, and $50 \mathrm{ug} / \mathrm{mL}$ zeocin, was used for screening of transformants [4, 35].

\section{Molecular cloning and plasmid construction}

The zeocin expression cassette was amplified from the pGZC-1 [4] with the primers 0426-3F and 0426-3R. The tubulin promoter, the actin terminator, and homologous fragments $18 \mathrm{~S} 1$ and $18 \mathrm{~S} 2$ were amplified from Aurantiochytrium genome with primer pair 194F/194R, 196F/196R, 191F/191R and 192F/192R, respectively. pfaE gene was amplified from S. japonica strain KCTC 22435 genome with primers $195 \mathrm{~F}$ and 195R. The pfaE expression cassette was assembled by the overlap PCR, and then ligated to the zeocin expression cassette and 18S1 and $18 \mathrm{~S} 2$ by overlap PCR (Additional file 1). To construct the $p p t \_a$ expression cassette, the $p p t a$ gene was amplified from Aurantiochytrium genome with primers 197F and $197 \mathrm{R}$ which contains the $2 \mathrm{~A}$ sequence. The plasmid vector was amplified from pGZC-1 with primers $198 \mathrm{~F}$ and 198R, and then, ppt_a gene was ligated to plasmid vector by seamless clone method (Additional file 4). The primer pair $191 \mathrm{~F} / 192 \mathrm{R}$ was used to amplify the line fragments for expression of $p f a E$ and $p p t \_a$, and then electrotransformation of Aurantiochytrium sp. SD116 was performed according to reported protocol [4].

pACYC Duet- 1 and pET Duet- 1 were used to express $\operatorname{orf} A$, orfB, orfC, $p f a E$, and $p p t_{-} a$. orfA, orfB, orfC, and ppt_a were amplified from Aurantiochytrium genome with primer pairs 1911-1F/1911-1R, 1911-2F/1911-2R, 1911-3F/1911-3R, and 1911-4F/1911-4R, respectively. $p f a E$ was amplified from $S$. japonica genome with primers 1911-5F and 1911-5R. The plasmid pET Duet-1 was digested with BamH I and Not I, and then ligated with orfC by seamless clone method to get pET-D-orfC. The plasmid pET-D-orfC was digested with Nde I and ligated with $\operatorname{orf} B$ to generate the plasmid $\mathrm{pET}$-D-orfC-orfB. The plasmid pACYC Duet-1 was digested with Nde I and Kpn $\mathrm{I}$ and then ligated with $p f a E$ by seamless clone method to generate pACYC-D-pfaE. The plasmids pACYC-DpfaE and pACYC Duet-1 were digested with BamH I and
Not I and then ligated with $\operatorname{orf} A$ to generate the plasmid pACYC-D-pfaE-orfA and pACYC-D-orfA, respectively. The plasmid pACYC-D-orfA was digested with Nde I and ligated with $p p t \_a$ by seamless clone method to generate the plasmid pACYC-D-orfA-ppt_a. pET-D-orfCorfB was co-transformed in E. coli BL21 with plasmids pACYC-D-orfA, pACYC-D-pfaE-orfA, and pACYC-DorfA-ppt_a, respectively, to generate the recombinant strains.

\section{Quantitative real-time PCR (qRT-PCR) analysis}

The total RNA was isolated using the Trizol reagent (Thermo Scientific) and miRNeasy Mini Kit (QIAGEN, Germany), and subsequent synthesis of cDNA was achieved using a Revert Aid First Strand cDNA Synthesis Kit (Thermo Scientific). The cDNA was used as the template for qRT-PCR analysis with the primers listed in Additional file 8. PCR was performed using a FastStart Universal SYBR Green Master (ROX). 18S rRNA was used as an internal control to normalize the expression levels. The relative transcription level was calculated using the $2^{-\Delta \Delta C t}$ method.

\section{Growth and biomass determination}

The biomass was expressed as DCW. $5 \mathrm{~mL}$ samples were collected and centrifuged at $9000 \mathrm{~g}$ at $4{ }^{\circ} \mathrm{C}$ for $5 \mathrm{~min}$, and then, it was determined by freeze-drying to constant weight at $-50{ }^{\circ} \mathrm{C}$.

\section{Lipid extraction and fatty acid composition analysis}

The total lipid was extracted using a combination of chloroform and methanol $(2: 1, \mathrm{v} / \mathrm{v})$, and weighed according to our previous report [4]. Then, the lipid was dissolved in chloroform and transformed to the fatty acid methyl esters (FAMEs) by using the method of Cui et al. [4].

FAMEs were analyzed by an Agilent 7890B gas chromatograph coupled with 5975C mass spectrometry. Separation was achieved on an HP-INNOWAX (30 $\mathrm{m} \times 0.25 \mathrm{~mm}$ i.d., $0.25 \mu \mathrm{m})$ capillary column with helium as the carrier gas at a constant flow rate of $1.0 \mathrm{~mL} / \mathrm{min}$. The GC temperature programming was set at $100{ }^{\circ} \mathrm{C}$ for $1 \mathrm{~min}$, then increased to $250{ }^{\circ} \mathrm{C}$ with increments of $10{ }^{\circ} \mathrm{C} / \mathrm{min}$, and held for $10 \mathrm{~min}$. The temperature of injection, transfer line, and ion source were 250 , 280 , and $250{ }^{\circ} \mathrm{C}$, respectively. The mass scan range was $50-800 \mathrm{~m} / \mathrm{z}$ and selected ion mode $(\mathrm{m} / \mathrm{z} 79.1)$ for quantitative analysis. FAMEs were identified by comparing mass spectra of the products with those of authentic ones and by utilizing National Institute of Standards and Technology (NIST) mass spectral library. 


\section{Calculation and statistical analysis}

All data are the means of three replicates and reported as the mean $\pm \mathrm{SE}$. The significance of differences $(p<0.05$, $p<0.01$ ) was assessed using a $t$ test.

\section{Supplementary information}

Supplementary information accompanies this paper at https://doi. org/10.1186/s13068-020-01793-x.

Additional file 1: Fig. S1. Scheme for expression of pfaE gene into Aurantiochytrium sp. SD116.

Additional file 2: Fig. S2. Genomic PCR detection.

Additional file 3: Table S1. Fatty acid profiles in strains SD1 16 and SD116::PfaE.

Additional file 4: Fig. S3. Alignment of PfaE with the putative PPTases from Aurantiochytrium sp. SD116 (PPT_A). Amino acid residues corresponding to core sequences of $\mathrm{P} 1 \mathrm{a}, \mathrm{P} 1 \mathrm{~b}, \mathrm{P} 2$, and $\mathrm{P} 3$ domains are underlined. PfaE_S from S. oneidensis, PfaE_M from M. marina, PPT_M from A. limacinum ATCC MYA-1381, PPT_H from $\mathrm{H}$. fermentalgiana.

Additional file 5: Fig. S4. Scheme for expression of ppt_a gene into Aurantiochytrium sp. SD116.

Additional file 6: Fig. S5. Genomic PCR detection.

Additional file 7: Fig. S6. The relative transcription levels of the fatty acid synthesis genes in transformant containing empty plasmid.

Additional file 8: Table S2. Primers used in this work.

\section{Abbreviations}

PPTase: phosphopantetheinyl transferase; ACP: acyl-carrier protein; PUFAs: polyunsaturated fatty acids; PKS: polyketide synthase; DHA: docosahexaenoic acid; EPA: eicosapentaenoic acid; KS: $\beta$-ketoacyl synthase; CLF: chain length factor; MAT: malonyl-CoA transacylase; KR: ketoacyl reductase; AT: acyltransferase; ER: enoyl reductase; DH: dehydratase; TFA: total fatty acid; FAS: fatty acid synthase; SFAs: saturated fatty acids; DPA: docosapentaenoic acid; qRT-PCR: quantitative real-time PCR; FAMEs: fatty acid methyl esters; DCW: dry cell weight.

\section{Acknowledgements}

We would like to thank HD Zhang, and YG Feng for their technical assistance. We would also like to thank prof. Yandu Lu for editing the English text of a draft of this manuscript.

\section{Authors' contributions}

QC and XS conceived the study. SW, CL, and ZW are responsible for the construction of mutant strains. WW and XS carried out the fermentation experiments. SW and XS performed the data analysis. SW and XS wrote the paper. All authors read and approved the final manuscript.

\section{Funding}

This work is supported by the National Key Research and Development Program (2019YFD0901904, 2016YFA0601400), Major Program of Shandong Province Natural Science Foundation (ZR2018ZB0208), Key Research and Development Program of Shandong Province (2019GHY112006, 2019YYSP003), the Key Deployment Project of Centre for Ocean Mega-Research of Science, Chinese academy of science (COMS2019J07), Qingdao source innovation program applied basic research project (18-2-2-50-jch), and QIBEBT (Grant: QIBEBT 1201933). This study is supported by Dalian National Laboratory For Clean Energy (DNL), CAS, and is a contribution to the international IMBER project.

\section{Availability of data and materials}

All data generated or analyzed during this study are included in this published article and its Additional files 1, 2, 3, 4, 5, 6, 7, 8 .

\section{Ethics approval and consent to participate} Not applicable.

\section{Consent for publication}

Not applicable.

\section{Competing interests}

The authors declare that they have no competing interests.

\section{Author details}

${ }^{1}$ CAS Key Laboratory of Biofuels, Shandong Provincial Key Laboratory of Energy Genetics, Shandong Engineering Laboratory of Single Cell Oil, Qingdao Engineering Laboratory of Single Cell Oil, Qingdao Institute of Bioenergy and Bioprocess Technology, Chinese Academy of Sciences, No.189 Songling Road, Laoshan District, Qingdao 266101, Shandong, China. ${ }^{2}$ University of Chinese Academy of Sciences, Beijing 100049, China.

Received: 30 May 2020 Accepted: 22 August 2020

Published online: 31 August 2020

\section{References}

1. Swanson D, Block R, Mousa SA. Omega-3 fatty acids EPA and DHA: health benefits throughout life. Adv Nutr. 2012;3(1):1-7.

2. Rogers LK, Valentine CJ, Keim SA. DHA supplementation: current implications in pregnancy and childhood. Pharmacol Res. 2013;70(1):13-9.

3. Moi IM, Leow ATC, Ali MSM, Rahman R, Salleh AB, Sabri S. Polyunsaturated fatty acids in marine bacteria and strategies to enhance their production. Appl Microbiol Biotechnol. 2018;102(14):5811-26.

4. Cui G-Z, Ma Z, Liu Y-J, Feng Y, Sun Z, Cheng Y, Song X, Cui Q. Overexpression of glucose-6-phosphate dehydrogenase enhanced the polyunsaturated fatty acid composition of Aurantiochytrium sp. SD116. Algal Res. 2016;19:138-45.

5. Gong Y, Wan X, Jiang M, Hu C, Hu H, Huang F. Metabolic engineering of microorganisms to produce omega-3 very long-chain polyunsaturated fatty acids. Prog Lipid Res. 2014;56C:19-35.

6. Park W-K, Moon M, Shin S-E, Cho JM, Suh WI, Chang YK, Lee B. Economical DHA (Docosahexaenoic acid) production from Aurantiochytrium sp KRS101 using orange peel extract and low cost nitrogen sources. Algal Research. 2018;29:71-9.

7. Dellero Y, Cagnac O, Rose S, Seddiki K, Cussac M, Morabito C, Lupette J, Aiese Cigliano R, Sanseverino W, Kuntz M, et al. Proposal of a new thraustochytrid genus Hondaea gen nov and comparison of its lipid dynamics with the closely related pseudo-cryptic genus Aurantiochytrium. Algal Research. 2018:35:125-41.

8. Morabito C, Bournaud C, Maes C, Schuler M, Aiese Cigliano R, Dellero Y, Marechal E, Amato A, Rebeille F. The lipid metabolism in thraustochytrids. Prog Lipid Res. 2019;76:101007.

9. Wallis JG, Watts JL, Browse J. Polyunsaturated fatty acid synthesis: what will they think of next? Trends Biochem Sci. 2002;27(9):467.

10. Napier JA. Plumbing the depths of PUFA biosynthesis: a novel polyketide synthase-like pathway from marine organisms. Trends Plant Sci. 2002;7(2):51-4.

11. Metz JG, Roessler P, Facciotti D, Levering C, Dittrich F, Lassner M, Valentine R, Lardizabal K, Domergue F, Yamada A, et al. Production of polyunsaturated fatty acids by polyketide synthases in both prokaryotes and eukaryotes. Science. 2001;293(5528):290-3.

12. Hayashi S, Naka M, Ikeuchi K, Ohtsuka M, Kobayashi K, Satoh Y, Ogasawara Y, Maruyama C, Hamano Y, Ujihara T, et al. Control mechanism for carbon chain length in polyunsaturated fatty acid synthases. Angewandte Chemie. 2019;12:32.

13. Yoshida K, Hashimoto M, Hori R, Adachi T, Okuyama H, Orikasa Y, Nagamine T, Shimizu S, Ueno A, Morita N. Bacterial long-chain polyunsaturated fatty acids: their biosynthetic genes, functions, and practical use. Marine Drugs. 2016;14:5.

14. Jiang H, Zirkle R, Metz JG, Braun L, Richter L, Van Lanen SG, Shen B. The role of tandem acyl carrier protein domains in polyunsaturated fatty acid biosynthesis. J Am Chem Soc. 2008;130(20):6336-7.

15. Okuyama H, Orikasa Y, Nishida T, Watanabe K, Morita N. Bacterial genes responsible for the biosynthesis of eicosapentaenoic and docosahexaenoic acids and their heterologous expression. Appl Environ Microbiol. 2007;73(3):665-70. 
16. Beld J, Sonnenschein EC, Vickery CR, Noel JP, Burkart MD. The phosphopantetheinyl transferases: catalysis of a post-translational modification crucial for life. Natural Product Rep. 2014;31(1):61-108.

17. Peng YF, Chen WC, Xiao K, Xu L, Wang L, Wan X. DHA Production in Escherichia coli by expressing reconstituted key genes of polyketide synthase pathway from marine bacteria. PLoS ONE. 2016;11(9):e0162861.

18. Wu J, Kim KS, Sung NC, Kim CH, Lee YC. Isolation and characterization of Shewanella oneidensis WL-7 capable of decolorizing azo dye Reactive Black 5. J Gen Appl Microbiol. 2009;55(1):51-5.

19. Orikasa Y, Nishida T, Hase A, Watanabe K, Morita N, Okuyama H. A phosphopantetheinyl transferase gene essential for biosynthesis of $n-3$ polyunsaturated fatty acids from Moritella marina strain MP-1. FEBS Lett. 2006;580(18):4423-9.

20. Meesapyodsuk D, Qiu X. Biosynthetic mechanism of very long chain polyunsaturated fatty acids in Thraustochytrium sp 26185. J Lipid Res. 2016;57(10):1854-64.

21. Weissman KJ, Hong H, Oliynyk M, Siskos AP, Leadlay PF. Identification of a phosphopantetheinyl transferase for erythromycin biosynthesis in Saccharopolyspora erythraea. Chembiochem. 2004;5(1):116-25.

22. Gross F, Gottschalk D, Muller R. Posttranslational modification of myxobacterial carrier protein domains in Pseudomonas sp by an intrinsic phosphopantetheinyl transferase. Appl Microbiol Biotechnol. 2005;68(1):66-74

23. Gao M, Song X, Feng Y, Li W, Cui Q. Isolation and characterization of Aurantiochytrium species: high docosahexaenoic acid (DHA) production by the newly isolated microalga, Aurantiochytrium sp SD116. J Oleo Sci. 2013:62(3):143-51.

24. Li J, Liu R, Chang G, Li X, Chang M, Liu Y, Jin Q, Wang X. A strategy for the highly efficient production of docosahexaenoic acid by Aurantiochytrium limacinum SR21 using glucose and glycerol as the mixed carbon sources. Bioresour Technol. 2015;177:51-7.

25. Park S, Kim K, Han S-I, Kim EJ, Choi Y-E. Organic solvent-free lipid extraction from wet Aurantiochytrium sp biomass for co-production of biodiesel and value-added products. Applied Biological Chemistry. 2017;60(2):101-8.

26. Wang F, Bi Y, Diao J, Lv M, Cui J, Chen L, Zhang W: Metabolic engineering to enhance biosynthesis of both docosahexaenoic acid and odd-chain fatty acids in Schizochytrium sp. S31. Biotechnology for biofuels 2019, $12: 141$.

27. Song X, Tan Y, Liu Y, Zhang J, Liu G, Feng Y, Cui Q. Different impacts of short-chain fatty acids on saturated and polyunsaturated fatty acid biosynthesis in Aurantiochytrium sp SD116. J Agric Food Chem. 2013;61(41):9876-81.

28. Sun XM, Ren LJ, Zhao QY, Ji XJ, Huang H. Enhancement of lipid accumulation in microalgae by metabolic engineering. Biochimica Biophysica Acta Mol Cell Biol Lipids. 2018;98:2.

29. Nishida T, Orikasa Y, Ito Y, Yu R, Yamada A, Watanabe K, Okuyama H. Escherichia coli engineered to produce eicosapentaenoic acid becomes resistant against oxidative damages. FEBS Lett. 2006;580(11):2731-5.

30. Orikasa Y, Tanaka M, Sugihara S, Hori R, Nishida T, Ueno A, Morita N, Yano Y, Yamamoto K, Shibahara A, et al. pfaB products determine the molecular species produced in bacterial polyunsaturated fatty acid biosynthesis. FEMS Microbiol Lett. 2009;295(2):170-6.

31. Reuter K, Mofid MR, Marahiel MA, Ficner R. Crystal structure of the surfactin synthetase-activating enzyme sfp: a prototype of the 4'-phosphopantetheinyl transferase superfamily. EMBO J. 1999;18(23):6823-31.

32. Chirgadze NY, Briggs SL, McAllister KA, Fischl AS, Zhao G. Crystal structure of Streptococcus pneumoniae acyl carrier protein synthase: an essential enzyme in bacterial fatty acid biosynthesis. EMBO J. 2000;19(20):5281-7.

33. Omura $\mathrm{S}$. The antibiotic cerulenin, a novel tool for biochemistry as an inhibitor of fatty acid synthesis. Bacteriol Rev. 1976;40(3):681-97.

34. Hauvermale A, Kuner J, Rosenzweig B, Guerra D, Diltz S, Metz JG. Fatty acid production in Schizochytrium sp: Involvement of a polyunsaturated fatty acid synthase and a type I fatty acid synthase. Lipids. 2006;41(8):739-47.

35. Cui G, Wang Z, Hong W, Liu Y-J, Chen Z, Cui Q, Song X. Enhancing tricarboxylate transportation-related NADPH generation to improve biodiesel production by Aurantiochytrium. Algal Res. 2019;40:101505.

\section{Publisher's Note}

Springer Nature remains neutral with regard to jurisdictional claims in published maps and institutional affiliations.
Ready to submit your research? Choose BMC and benefit from:

- fast, convenient online submission

- thorough peer review by experienced researchers in your field

- rapid publication on acceptance

- support for research data, including large and complex data types

- gold Open Access which fosters wider collaboration and increased citations

- maximum visibility for your research: over 100M website views per year

At $\mathrm{BMC}$, research is always in progress.

Learn more biomedcentral.com/submissions 